\title{
LOS ADVERBIOS DE ORIGEN NOMINAL Y EL PROBLEMA DE LA HETERÓCLISIS $*_{-r} / *_{-n}$
}

This paper attempts to explain the form of the Indocuropean prepositional adverbs ending in $*_{-r} /-n$, their dialectal distribution (basically $*_{-n}$ in Anatolian, $*_{-r}$ in other Indoeuropean languages) and their relationship to the $-r /-n$ noun stems.

1. El propósito de este trabajo es estudiar aquellos advs. ie. de origen nominal que no conocen formas flexivas en ninguna lengua, es decir, los llamados «advs. preposicionales»; y se contemplarán sólo en tanto en cuanto alargados por los sufs. ${ }_{-} r y^{*}-n$. Por tanto, se entiende que quedan automáticamente excluidos usos adverbiales de sustantivos existentes sincrónicamente en una lengua (ai. áhar-áhar 'de día en día'), locs. adesinenciales (áhan), advs. con suf. *-ter (lat. inter) y advs. de origen pronominal (gót. hwa-r, lat. $c u-r$, ai. tár-hi). El hecho de que algunas de las raíces tratadas presenten derivados pronominales o adjetivales supone tan sólo una tematización secundaria de aquéllos en su forma básica, que de por si es un adverbio y funciona frecuentemente como tal en todas las ramas: me refiero a ${ }^{*} \mathrm{H}_{2}$ eu-o-, ${ }^{*} \mathrm{H}_{1}$ en-o-, ${ }^{*}$ per $\mathrm{H}_{3}-\mathrm{o}$-. Este fenómeno no toca en absoluto a la presente discusión. Naturalmente, las raíces que se van a tratar presentan en las lenguas un conjunto altamente variado y complejo de finales adverbiales. La selección de $*_{-r}$ $y^{*}-n$ obedece a varias constataciones. La primera consiste en que, de los finales básicos conocidos para este subtipo de adverbio nominal, el «preposicional» (a saber, fundamentalmente y prescindiendo de la interpretación laringal de los finales vocálicos, ${ }_{-r}, *_{-} n, *_{-} i, *_{-} u, *_{-} a, *_{-},{ }^{*} *_{-} / \bar{o}, *_{-s}$ ) sólo dos de ellos, ${ }_{-r}$ y $*_{-n}$, son asimilables, mutatis mutandis, a uno de los tipos de la heteróclisis nominal, precisamente al tipo con diferencia más extendido en la familia indoeuropea. Otro tipo posible, $*_{-i} / *_{-} n$, tiene una extensión demasiado reducida: cuatro neutros en ai, y el tipo * poti-/*potn-, que sirve para oponer género y no caso, y que ha servido en báltico de modelo para dos ejemplos más !

\footnotetext{
' Cf. un desarrollo particular de estos datos en Álvarez-Pedrosa, 1988.
} 
El segundo móvil para la combinación de $*_{-r} / *_{-n} n$ en este estudio es su aparente paralelismo. En efecto, podrá objetarse a todo lo anterior que los advs. en $*_{-} r$ y en ${ }^{*}-n$ resultan asimilables, en todo caso, a los tipos nominales res. pectivos en ${ }^{*}-r \mathrm{y}^{*}-n$. Pero entonces habrá que explicar también por qué desde el punto de vista de la formación de palabras aparecen sobre las mismas bases, lo que apunta a que su formación y expansión fue aproximadamente contemporánea. Y en ese caso, parece, por una parte, inútil alargar los advs. preposicionales según varios temas diferentes si no hubo nunca relación de complementariedad funcional entre $*_{-r} \mathrm{y}^{*_{-} n}$. Y, por otra parte, nada tienen de especial los temas en $*_{-r}$ y en $*_{-n}$ nominales para justificar este fenómeno: p. ej., no hay resto ninguno entre estos advs. de temas en oclusiva, o en $*_{-} l$, como tampoco hay, curiosamente, formas derivadas ni «flexionadas» que tengan como base ninguno de estos advs. preposicionales sufijados.

2. Pasemos al análisis pormenorizado del material, que se agrupa por raíces.

2.1. $* \mathrm{H}_{2} \mathrm{eu}$-.

Ai. áva 'hacia abajo, hacia fuera', aváh ( $r$ sólo en $R V \mathrm{I}, 133,6)$, 'hacia abajo', *arvä en arvañc- 'dirigido hacia abajo'. Adj. derivado ánara- 'el de abajo' / nav. ava 'sin embargo', av., ap. ava/-ä 'hacia' (+ ac.), nav. avard, aav. avard (Y. 29, 11) 'hacia abajo', nav. aora, aav. aora 'hacia abajo' '2. Formas emparentadas con suf. $-r$ en dialectos mir. y nir. Nyberg (1932, pp. 246-47 y ns., 260-61) parte de la alternancia en ie. de una serie de variantes sufijales $-r$,

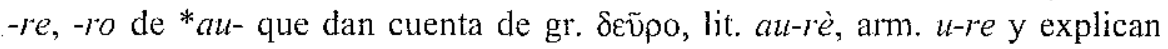

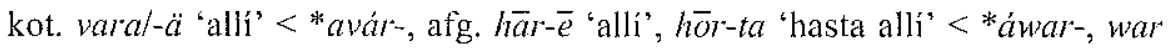
'a él' < *awár, kurdo $\bar{o} r-a /-d a, l-\bar{o} r-a$; Talahedäšk $\bar{a} r-\bar{a}$ 'allí' ${ }^{3}$. Benveniste

\footnotetext{
${ }^{2}$ Ap. aura en una de las inscrs. de Darío (Pers. e $3^{7}$, Weissback 1911, pp. 82-83) no es instr. de $a^{h} u r a-$ 'señor', que aparece siempre como $a^{h}$ uramazda-, sino un adv. de esta raiz. El sentido de la frase es patente si aurā significa 'hacia abajo': la traducción habitual de anrāa nirasătii es 'la bendición bajará a esta casa mediante Ahuramazda', pero puede ser literalmente 'la bendición bajará (nirasătii) hacia abajo (aura) a esta casa'. Así Hoffinann (1956, pp. 9-10), que hace corresponder ai. *arvālpáră con av. aor $\bar{a}-c \bar{a} / p a r a-c \bar{a}$. Wackernagel $(1918$, p. 393) retrotraia aora a avard bajo influjo de para $\bar{a}$; esto no es fónicamente posible, porque aor $\bar{a}$ no puede ser representación gráfica de *avarā. Tenemos pues una forma aria *aura 'hacia abajo, hacia aquí' que sirve igualmente para véd. *arvä < *a-vrá (en arvañc-) con una silabación que preservaba la identidad etimológica. Para $A I W$ (s. u. j. g. aora/a) aora es instr. sg. de *aora-, adj. cpvo. de ava.

${ }^{3}$ Hay casos de otros temas: kot. mara 'aqui', ttara 'alli'. La vocal final en las formas nir. es secundaria y representa un caso adverbial; $-a$ cn kurdo y $-\bar{a}$ cn Talahedäšk pueden reflejar un sust. mutilado; - $t a$ en afg. es posp. Que en fases posteriores al air. no haya desaparecido la consonante final podría revelar que habia una vocal final (aunque no necesariamente en el caso de $-r$ y sobre todo si la sílaba era tónica). Pero kot. $-r a l-a ̈$ parece reposar sobre un antiguo -ra, del tipo avára, imára, con lo que para Nyberg es equiparable a gr. $\delta \varepsilon \vec{u} \rho 0$.
} 
(1956, pp. 30-31) da una forma más: así como osét. deelce 'debajo' corresponde a av. $a \delta a i r i$, a su antónimo ucelce 'encima' le corresponde una base *arari, y no upairi, como se cree, que está en osét. bcel. Thordarson (1989, p. 470) ofrece para los étimos *avari, *adari osét. uael y dal. En resumen, existen en i.-ir, *avar, *avari y *aur.

It.: Existe un pron. pit. *uro- en u. uru 'illo', wres 'illis', etc. Cf. Brugmann (1904, p. 98), que lo considera procedente del pron. $-u$ - con formante $-r$ como lit. aurè.

Lit.: aùè, aurenaĩ, auriōn, auriõnai 'allí, de ahora en adelante' (diccionarios). BSLW (s.u. aua-) pone lit. aurè en relación con los pron. esl. e ir. derivados de esta raíz. Sin embargo, el vocalismo final plantea problemas. Nyberg (1932, p. 260) afirma que contiene un suf. *-re como arm. ure y que

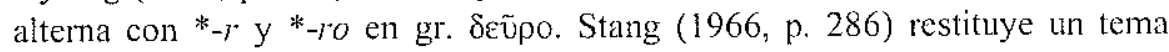
*avar- alargado, que además habría sufrido sincopación de -a- en silaba medial, y cita (n. 2) una forma lit. oriental varakui. En cambio Januschas (1962, p. 35) cree que se trata de un loc. en *en por causa de almenăi, a su vez adverbialización común lit. en -ăl, sobre la base *auren. De hecho, BSLW (s. 4. aua-) menciona tanto aurè como aurén. En cuanto a auriōn[-ai], Januschas cree que están constituidas como namõn (que pertenece a una serie de advs. ilativos construidos con la posp. $-n$ ) y relacionadas con gr. $\alpha u$ pıov 'mañana'. La teoría de Januschas es verosímil en lo que respecta a ambas recaracterizaciones; pero la conexión con gr. aüplov es claramente incorrecta, ya que esta palabra se relaciona con lit. aušrà 'aurora'. Lo más probable es que tengamos en *auri una base *aur rehecha por $-i$. Hemos visto que en i.-ir., junto a la base *avar, existen restos de un adv. osét. ucelce $<*$ avari. Estas formas contienen el suf. *-er- en g. P; plit. *aur y *auri son sus equivalentes con suf. $\emptyset$.

Het.: $a w a n^{4}$. Melchert $(1984$, pp. 22, 61) parte de * $a w-V n$, que compara con ai. áva, lat. au-, luvc. $a w-i$. Opina que la palabra empieza por pie. ${ }^{*} a-$, pues de tratarse de ${ }^{*} H_{2}$ tendriamos ante $-w$ - $h w$ - como en huis- 'vivir' $<{ }^{*} H_{2}$ wesHamp (1965, pp. 125-26) ve aquí, como en $u^{-}$, we-, wa-, y ai. áva, lat. a $a u^{-}$, una base $* h w-$. *h- $\left(H_{4}\right)$ no deja restos en anat, pero sí en alb., cf. vac. $/ \Gamma i h /$, sof. /hiin/, etc. 'yo entro' < palb. *hinvo 'entro'. Si het. awan tiene $-n$ antigua, y no una terminación, es posible dividir la forma alb. en *hwn- más el productivo suf. verbal *-y-/-i-; quizá hay aquí huellas remodeladas de la forma que se encuentra en het. unnai 'conduce hacia acá'.

\footnotetext{
${ }^{4}$ Sin existencia autonoma; aparece en las expresiones awan arha, que indica separación y awan katta 'hacia abajo'; según la escucla tradicional como prev., adv. o posp. de abl. (Zuntz. 1936, pp. 44-45).
} 
A veces se han relacionado las terminaciones en $*_{-r}$ y $*_{-n} /{ }^{*}-m$. Sturtevant (1931) parte de una base apofónica **awe $(m)$, de donde indo-het. **au-, aw n, we-, wo- 'hacia aquí abajo', con 'especial referencia a la bajada de los cuerpos celestes'. Ai. ava puede pues identificarse con het. awan sobre la base de indo-het. e ie. ${ }^{*} a w-m$. Polomé (1952, p. 1043) reconstruye ${ }^{*} H_{2 e} w e ́ r$ en ai. avár que compara con $* H_{2 e}$ wón en het. awan. HED relaciona con awan lit. aurè,

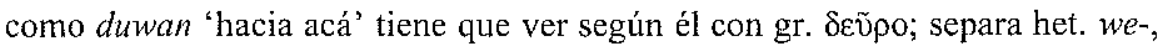
$u$-, ai. áva, avár, lat. au-. Dunkel (1988a, pp. 113-121) parte de una forma doble original *awo/we-: *aw-. *aw $<$ *awu está en un adj. *aw-ro- (frente a *aworo- en ai. ávara-) que subyace a lit. aurè, av. aoral-a y *arwa-. El modelo *apu indica que se trata de un alomorfo supletivo. Parte para het. awan de *aw-en, forma según él más probable que *awo-n.

Parece claro que en het. awan se encuentra la variante $-n$ de un esquema de heteróclisis cuyo tema en $-r$ está en lit. aur-, i.-ir. *avar, *aur-, it. ur-. De igual modo se explica ai. ava como ie. *aw-n. Yo reconstruyo *au-como ${ }^{*} \mathrm{H}_{2} \mathrm{eu}$ - La primera dificultad estriba en el doble g. P que parece exigir al menos i.-ir. ${ }^{*}$ an-ar $<{ }^{*} H_{2} e u$-er. La solución consiste en reconstruir un tema ${ }^{*} \mathrm{H}_{2} e u H$-, que se deduce del testimonio de lat. y anat. we-, cuyo g. $\emptyset$ vocalizado estaría en het. $w a$ - (?), gr. (glosa) ỏ $\alpha \vartheta$ r. Esto no es imprescindible para *aur, *auri. O bien habría que pensar en una discutible vocalización $* H->a$ - en inicial en i.-ir. En tal caso habría que reconstruir simplemente una alternancia ${ }^{*} \mathrm{H}_{2} \mathrm{eu}-\mathrm{r} /{ }^{*} \mathrm{H}_{2} \mathrm{eu}-n:{ }^{*} \mathrm{H}_{2} u-e r /{ }^{*} \mathrm{H}_{2} u$-en; la vocalización de $\mathrm{H}$ - en este último étimo daría razón de la inexistencia de grupo inicial het. $h w$ -

\section{2. $* H_{1}$ et-.}

It. El pron. 'el otro' es en lat. alter. En u., en cambio, encontramos una base etro- en etrama 'ad alteram', etraf 'alteras', etru 'altero', etc. Tiene correspondencias en nav. atära-, aesl. jetern, y lat. cēterus 'el restante' si es que < *ce(i)-eteros. Hamp (1965, p. 138) reconstruye * $H_{1} e ́ t(e) r$-, y compara adicionalmente alb. $t$-jetër. Podemos añadir el pron. recíproco arm. irears, probablemente de *eteros-eterom (Rasmussen 1987).

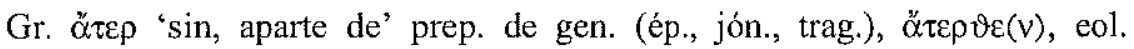

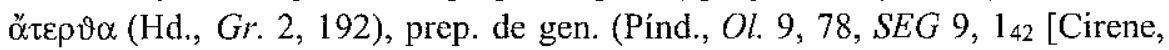

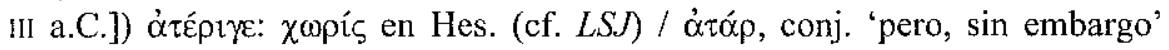
(ép., át.). ớ $\varepsilon \rho$ ha dado lugar a discrepancias sobre su origen. Algunos autores han propuesto ver en $\alpha$ - el descendiente de ie. * $s n$ - ${ }^{5}$. En cambio Beekes (1982, p. 207) reconstruye *sm-ter, de *sem- 'uno'. Meier-Brügger (1988, pp. 13741) señala que, caso de que $\alpha$ $\alpha \varepsilon \rho$ proceda de ${ }^{*}$ sn-tér, debe, por el acento y la

\footnotetext{
5 Así Bugge (1879, pp. 120-21), Kretschmer (1892, p. 350), que identifica ö sanutár y as. sundir como procedentes de *snlér, GEW, DELG.
} 


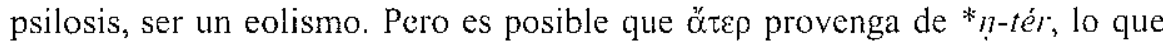
elimina el problema de la falta de aspiración. En gr. se habría hecho común el valor abl. que aparece en restos lat. (inter-ficere) y aav. Sin embargo, lo

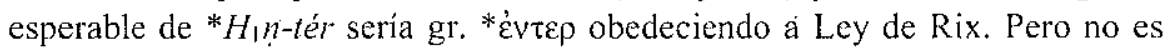
necesario, tal vez, suponer aquí una $H$-, sino quizá ${ }^{*}$ en $/{ }^{*} n$ como en el caso de alfa privans. Ven. a.tra/antrā y e.n.to.l/entol representarían *nt-/*ent-, puesto que, admitida la triple representación de laringal en lat., y si esto es extensible al it., tanto ${ }^{*} H_{1} n t$ - como ${ }^{*} H_{1}$ ent- hubiesen dado ent-. El autor concluye que no es seguro si $\alpha$ $\tau \varepsilon \rho$ representa $*_{s m-t e ́ r}$ o ${ }_{n}$-tér, pero se inclina por lo primero. Ya Meillet (1902, pp. 153-54) proponía *n-ter ${ }^{6}$.

Pasemos a la cuestión planteada por $\dot{\alpha} \tau \alpha \dot{\alpha} \rho . \operatorname{DELG}$ (s.u. $\dot{\alpha} \tau \alpha \dot{\alpha} \rho)$ entiende que

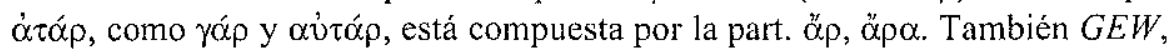
que compara gót. $a b$ - $[b a n]$, lat. at. Por otro lado, se ha propuesto en ocasiones

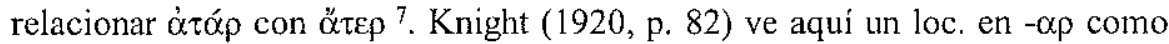

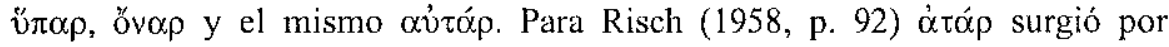
abreviación de $\alpha$ $\tau$ $\alpha$ (responsable de esta idea es realmente Düntzer). Por

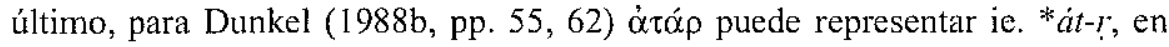
paralelo a lat. at-que y donde $r$ es una conj., o incluso ${ }^{*}$ át- $\mathrm{H}_{2} \mathrm{O}-\mathrm{r}$ (ai. átha $=$ $* a ́ t-\mathrm{H}_{2} \mathrm{O}$ ) salvo porque quizá ${ }^{*}{ }_{-} H_{2} \mathrm{O}>-0$. Adopta una propuesta de Schindler

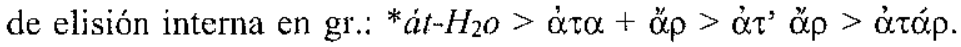

En principio, a pesar de sus inconvenientes fonéticos, una forma $*_{\text {snter }}$ parece dar cuenta de ǒ́tep. Sin embargo, a raíz de una observación detenida de los hechos que conciernen a la raíz $* s(e) n$ - en las demás lenguas, encuentro a esta reconstrucción una dificultad adicional. $Y$ es que, en general, hay evidencia de una $-H$ - que sucede a la nasal o un resultado que admite esta posibilidad en lenguas que no distinguen sonante interconsonántica de «sonante

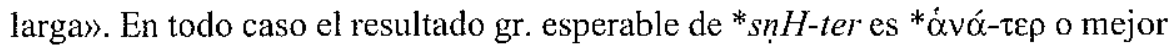

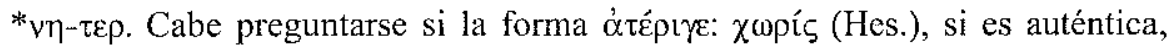

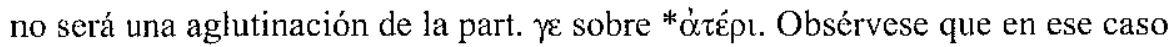

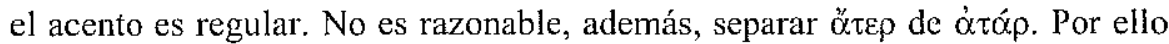

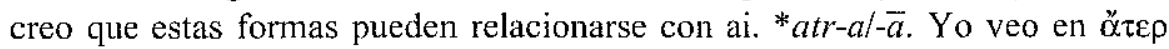
una relación $\emptyset / \mathrm{P} * H_{1} t e ́ r$, en $\dot{\alpha} \tau \dot{\alpha} \rho$ un $\emptyset / \emptyset{ }^{*} H_{1} t r$, bajo el supuesto, compartido por otros autores, de que existen dos raices sinónimas ${ }^{*} H_{1} t-{ }^{*} H_{2} t-$, o bien

\footnotetext{
${ }^{6}$ El problema suscitado por un 1 . $^{\text {tr }}$ término ${ }^{*} n$-, aparentemente $\sin H$ - desde el punto de vista mencionado, se agudiza porque en gr. existen compuestos en que alternan $\dot{\alpha}-/ \dot{\varepsilon} v-$, dificiles de interpretar por la confluencia fónica con $\dot{\alpha}$ - privans y (en dial. psilóticos) *sm-.

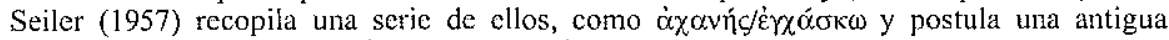
distribución ${ }^{*} e ́ n$ indep. $/^{*} n_{0}{ }^{\prime}$ procl.: cf. gr. Ěvı, ai, $n i-d \dot{a}-<{ }^{*} n i-z d-\dot{o}-$.

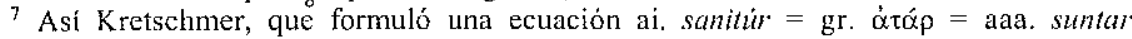
$<*$ séntr, o Bugge $\left(1879\right.$, p. 121). En realidad, ${ }^{*} s n-t r$ sería la única protoforma posible, con el defecto, como ǒxep, de la ausencia de aspiración.
} 
sobre la base de que el resultado inicial de $* H_{1}--\mathrm{C}$ es vacilante. El sentido separativo es común en las correspondencias de *et-. Por otra parte, sólo hay evidencias de una forma *eter en el pron. *eteros, analizable como *eter-os o como *et-eros. La 1." posibilidad viene avalada, como veremos, por paralelos como *ener-os frente a *ener. En tal caso hay que pensar en un adv. * $H_{1}$ et $(H) e ́ r$. La 2. ${ }^{a} H$ - es postulable a la vista de *ata en lit. ata- 'a su vez, de vuelta, fuera', gót. ab-, aing. ced-, galo ata- en Ataazti, atha-en Athamallus, etc. y *eta en aing., as. ed-.

2.3. ${ }^{*} H_{1}(e) n-$.

It. Existe una base "ner en u. nertru, o. nertrak 'sinistro', que tienen correspondencias en germ. en aisl. nor $\gamma r$, etc. 'norte' $<* n r$. Por lo demás, existe un pasaje en alat. que puede mostrar huellas en esta lengua de un adv. ner. Se trata de la fórmula augural para la delimitación del templum recogida por Varrón (De ling. lat. 7,8 ) en que se dice ullaber arbos, quirquir est, quam me sentio dixisse, templum tescumque [f]esto sinistrum; ollaner arbos, quirquir est, quod me sentio dixisse, templum tescumque [f]esto dextrum. J. Schmidt (1892, pp. 416, 419) corrige la lectura de los mss., que es ullaber, ollaner, y leyendo olla-per (cf. topper, paulisper) traduce 'jener Baum, dessen Ort ich zwar nicht genau angeben kann, den ich aber ganz genau im Sinne habe, soll linkes Templum und Tescum sein'. En cambio Pighi (1941, pp. 255, 231), siguiendo la interpretación de Norden, ve en ollaber\%llaner un doblete constituido por dos suf. locs. -ber y -ner, siendo el $1 .^{*}$ *dwer y el $2 .^{\circ}$ ner. Tal vez debamos pensar aquí, como en germ., en una referencia directa a los puntos cardinales.

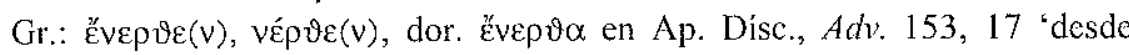
debajo, debajo', prep. de gen. 'bajo' (Hom.), 'desde debajo de' (Esq., Pers.

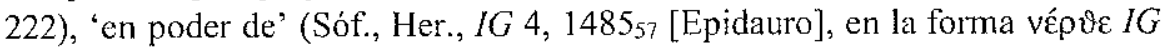
12 [2], 74b $\mathrm{b}_{21}$ [Mitilene, III a.C.]), adj. Ěvepor 'los que están bajo la tierra (muertos y divinidades infernales)' (Hom.).

Para (') vep-, IEW, p. 766 parte de *ner- 'debajo', y especula con una posible relación con *nei 'abajo'. Güntert $(1910$, p. 49) opina que existían al principio

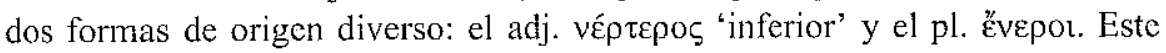
último procedía de *Ev más el suf. *-ero-, y quería decir 'los de dentro', o sea 'los muertos, que habitan el reino de las sombras'. Ambas palabras habrían

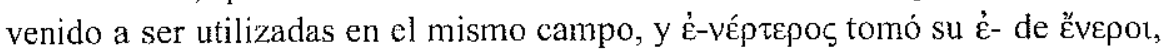

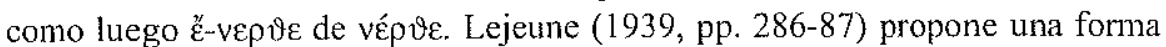
* $H_{1}$ ner, forma sufijada que sustituyó a *H $H_{1}$ en en su significado 'debajo', a su vez eliminada por la sinonimia con *H $H_{1}$ en 'dentro'.

Lenguas bált. Stang (1966, p. 286 y n. 2) ve una forma *anrè 'siehe da!' (cf. aurè), en la base de lit. oriental unrakkui, como ejemplo de un tema *anar-, con sincopación de - $a$ - en sílaba medial, como varãkui (cf. ${ }^{*} \mathrm{H}_{2} \mathrm{eu}$-). 
Het annan (?); luvc. anna(n), prep. 'bajo', luvj. *annan prep. 'bajo', lic. $\hat{e} n \hat{e}^{8}$. Laroche (1957, pp. 180-82) ha identificado las formas lic. y luv. Lic. ênê se observa en expresiones como ênê Periklehe xñtawata, que lee 'suboficial de Pericles'. Según el autor, el het. confunde en katta(n) dos órdenes de relaciones espaciales que distingue el luv.: el estático annan y cl dinámico, de movimiento dirigido, kata. Carruba identifica con las formas luv. y lic. het. annan, cuyo significado no está claro (cf. HEG s. u. annan). Gusmani (1963) objeta a Laroche que ênê nunca va escrito junto con xintawata y que se le opone el orden de palabras. Además, hay que preguntarse por qué tantos 'suboficiales' y ningún oficial. Mejor es suponer una fórmula de datación, concibiendo xñtawata como abstracto: ênê xñtawata = 'durante/bajo el gobicmo (de...)'. Xñtawata sería dat.-loc. regido por ênê.

Hemos visto documentada en it. y gr. una forma ${ }^{*}$ ner que puede retrotraerse sin problemas a ${ }^{*} H_{1} n e r$, con una variante $\emptyset$ en germ. ${ }^{*} H_{1} n r$. Este ${ }^{*} H_{1} n e r$ es idéntico a la base de gr. vep-. Por tanto, sólo queda por esclarecer gr. évep-. En principio, podría tratarse simplemente de * $H_{1}$ ner bajo la suposición de la triple representación de las laringales, al menos en inicial ante sonante y consonante (cf. gr. ג̇víp) ${ }^{9}$. O bien, atendiendo al paralelismo con *awer-, *eter-, habría que reconstruir *ener-, esto es $* H_{1}$ ener; para obviar el problema del $\mathrm{g}$. $\mathrm{P} / \mathrm{P}$ es necesario, como ya hemos visto, postular ${ }^{*} H_{1} e n(H)$ er. Pues bien, Hilmarsson ha propuesto la existencia de un adv. ${ }^{*} n(e) H_{1} i$ que yo, por su parentesco con el tema* $H_{i}$ en-, corrijo en $* H_{1} n(e) H_{i} i$ (Prósper 1993). Como en otras raíces, también en ésta el tema en $r$ sería un derivado sobre un antiguo tema en laringal. Anat. *anan 'debajo' parece estar en la misma relación con lat. -ner, gr. (દ) vep-, lit. *anr-, que anat. *awan frente a ic. *aur/*auer. *anan procederá seguramente de ${ }^{*} H_{1} n H_{1}$ en o $* H_{1}$ nen. Una evolución desde una forma cuya base fuera el g. $\mathrm{P} * H_{i}$ en- es improbable: esperariamos *enan.

En het. existe una forma tuwa 'lejos', que se pone en relación con una base

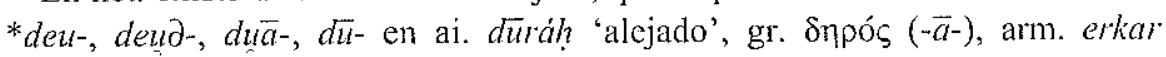
'mucho tiempo', erkain 'largo' (IEW, pp. 219-20). Al lado existe una forma muy parecida duwan en la expresión duwan...duwan 'hacia acá...hacia allá. $E H S$, p. 353, reseña que no tiene variante en $-a$, a menos que esté en relación con tuwa 'lejos'. Melchert (1984, p. 30) ve en tu-Lt-wa (/duwal) y $t u-w a-a-a n$ $(/ d w \bar{a} n /)$ dos casos de un nombre raíz *dweH ${ }_{2}$. Dwān sería un ac. *dweH $H_{2} m$,

\footnotetext{
${ }^{8}$ Ya Pedersen (1945, p. 65) rechazaba la interpretación de lic. ênê como '(ćl) era', referido al muerto como jefe militar de algún señor. La cópula no es necesaria, y ênê podría ser más bien un adv. 'antaño, hace tiempo'.

9 En ese caso, lit. or. unrakui (que podría ser una formación tipica de adv. $+* H_{3}(e) k^{\prime \prime}$ )

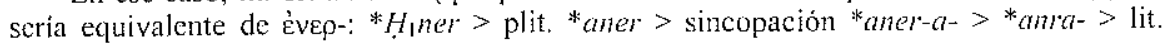
oriental thr $r(i-$.
} 
duwa no puede ser más que un loc. puro (con variante Lindeman) *duweH $\mathrm{H}_{2}>$ *dwwah $>*$ diw $\bar{a}>*$ diwa, sin evolución subsiguiente a -uma-por analogía de $d w$ - Dunkel (1988a, p. 109 y n. 10) separa tuwa- 'lejano' $<* d w e H_{2} m$ [isic!] de duwan 'hacia acá'. Duwan continúa ie. *dew-en, y pertenece a una cadena deíctica $* d e-w$ - (part. direct. + deíctico ${ }^{*} u$ ). gr. $\delta \varepsilon \tilde{\rho} \rho$ resulta de aquí por adición de $* r$ adverbial loc. Quizá la secuencia original sería $* d e-r-u$, con posterior distorsión del orden de constituyentes deícticos. Sobre duwan: $\delta \varepsilon v ̃ p o$ cf. también $H E D$ (s.u. awan).

Estos autores no han contado con un testimonio poco conocido. En lat. arc. existió un adv. encl. o suf. -ber, del que hablé antes. Pighi (1941) cita la expresión iterativa del Carmen Arvale sta berber 'quédate ahí mismo', y reconstruye * $d w e r$. En vista de lo cual se podría pensar que het. duwan no tiene, a juzgar por el significado, nada que ver con tuwa, y además constituye el

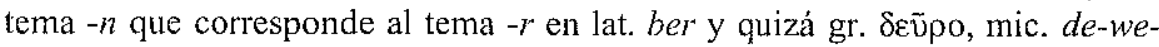
ro-. En ese caso, el significado original no habría podido ser 'hacia aquí'. Tal vez ćste era el tema que indicaba algo así como 'frontalidad', luego cercanía, finalmente aproximación a un punto determinado subjetivamente y expresado por el adv. mismo, como ocurre en los advs. pron. Mic. de-we-ro-contendría una base * dewer- con las caracteristicas de *awer, *eter, *ener. Y la explica-

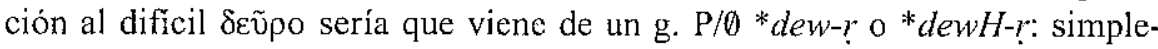
mente tendríamos en este adv., ya homérico, una vocalización de sonante del tipo eol./arc.-chip. o $\rho / \rho \circ$, proceda $\delta \varepsilon \tilde{p} \rho \circ$ o no de una metátesis de ${ }^{*} \delta \varepsilon F o \rho$.

2.4. ${ }^{*} \mathrm{H}_{1} \mathrm{ep}$-.

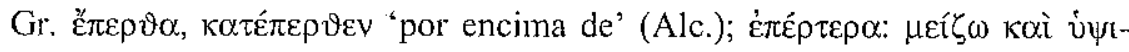
$\lambda o ́ \tau \varepsilon \rho \alpha$ (Hes.) pueden parecer refecciones basadas en la existencia de évep-

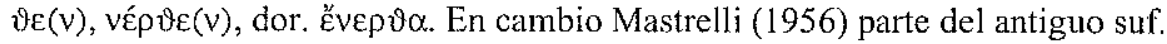
adverbial locativizante ${ }^{*}-e r$-, paralelo a *-ter-, y determina que estas formas y su correspondiente adj. ह̌ $\pi \varepsilon \rho o t$, que aparece en una inscripción eol. (IV-III a.C.) y que él contempla como adjetivación de ëлzep, provienen de *eper. Proporcionan apoyo para esta reconstrucción alb. épërë 'superior' y airl. iar 'después', formas que el autor hace también proceder de *eperos ${ }^{10}$. La consecuencia es que en este caso el eol. muestra cierta individualidad respecto al resto del gr. Es posible ver ع̌̃

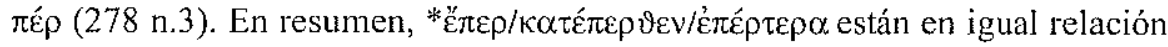

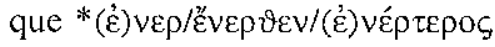

10 Hamp (1981, pp. 42-43) indica que alb. épër (ocasionalmente épërm), no puede tener *e- inicial, que daria *je-. Por tanto, la única construcción razonable sería *opi/i-ro-. Esto encaja con airl. $i a{ }^{*}$, iarm 'tras' < ${ }^{*} e p i-r o-m / *_{e p i-r o-(m-\eta)}$. Antes (1965, p. 136) relacionaba alb. épërë con gót. iup sobre una base *Hieru-. 
Así, غ̇лep- provendrá seguramente de ${ }^{*} H_{\uparrow}$ epHer, o bien ${ }^{*} H_{1}$ per. La posibilidad de una $-H$ - medial viene indicada una vez más por testimonios como toc. A pe (conj. pospuesta) 'también' < *péi, quizá en parte el prev. het. pe-de alejamiento del hablante.

\section{5. * ${ }^{*} \mathrm{H}_{2} \mathrm{ep}$ -}

I. -ir. Adj. ai., av., ap. ápara- "posterior' 11. Forma verbal vyapanenti (Udānagātha) $=$ pāli vapayanti (citadas en J. Schmidt 1885); nav. apąm 'tras esto, en lo sucesivo'. AIW (s. u. apam) pone apam en relación con ape. En GIPh se considera apqm como instr. en *ām. Creo que tanto ai. -apan- en vyapanenti como nav. apam (¿por *apan?) están en relación con ${ }^{*} H_{2} e p-$. El supvo. nav. apanōt $\partial m a-$ 'el más alto’ podría derivar directamente del adv. (¿con $-\bar{o}-$

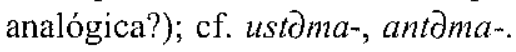

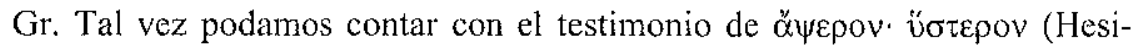

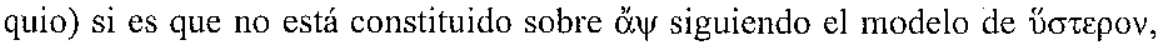
sino sobre una forma *aper (cf. Mastrelli 1956), que procedería de ${ }^{*} \mathrm{H}_{2}$ per:

Germ.: aaa. afar, avar 'de nuevo, de vuelta, pero, y', fi. afur, abur, avur, alemánico avur', bávaro afur, aisl. afar-, avar- 'muy', aur- pref. 'de nuevo, contra-', gót. afar prep., adv., perf. 'tras, después de'. G. Schmidt (1962, pp. 264-67) cree que gót., aaa. y aisl. afar reposan sobre *operom, y explica el vocalismo aaa. - a- por Brechung. Supone asimismo que aaa. abur, aisl. aur-proceden de ie. *apúr. A. M. Sturtevant (1927, pp. 142-44) menciona como prefs. intensivos aisl. afar , af $r^{*}$, of $r^{-}$. Afar es, según él, el cpvo. de af. Para diferenciar el adv. ofar del pref. intensivo ofar- éste se redujo a of $r$-, según el modelo de los intensivos en $-r$ $<i z$. A su vez, afi es analógico de ofr <*upor. Cree que aur-procede de *apr; como aaa. abur. Sin embargo, me parece más fácil ver en gót. afar, etc., ie. *apor, como Johansson $(1890$, p. 131) y como admite el mismo G. Schmidt en el caso de *upor* $>$ aaa. -ar. A su vez, *apor procede de * $\mathrm{H}_{2} \mathrm{epH}_{3} \mathrm{er}$ o ${ }^{*} \mathrm{H}_{2} \mathrm{pH}_{3} \mathrm{er}$. La existencia de $-H_{3}$ - medial, y por tanto de un paralelismo formacional con *awer, *eter, *ener, *eper, es evidente tanto por el final *-or como por la naturaleza de la base *apo, ${ }^{*} p \bar{o}$, remitible a ${ }^{*} \mathrm{H}_{2} \mathrm{peH}_{3}{ }^{12}$.

Lat. apud 'junto a' permanece inaclarado ${ }^{13}$. Al lado existe aput (DEL s. u. apud), así como una serie de formas dialectales con variaciones en el consonantismo final: son apor (en Festo 24, 12), apur (en territorio marso, cf. CIL

II Una forma poco clara es ai. punar. Para Pighi (1941, pp. 230-31), contendría *ner de

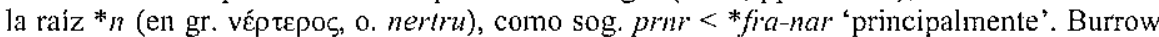
(1955, p. 277) incluye púnar entre las formas con el suf. -ar, empleado en la construcción de temas de sust. n., a cuyo loc. son iguales. G. Schmidt $(1962$, p. 260) piensa que * pur-ne $>$ *purna $>$ puinar.

12 Y cf, tal vez $\dot{\alpha} \pi \alpha-i=$ ónò (Empéd. 134, Dion. Perieg. 51), si no es analógico.

13 Según $L E W$ (s. us apud) es un partic. perf. act. *apuot 'que ha alcanzado' de apio, apiscor y por ello no aparece como adv. o prev.; su significado primitivo es 'en cercana 
1 25: apur finem). Es probable que pronunciaciones que parecen corruptas, como apor, apur, revelen el estado de cosas original, del que apud podría representar la interpretación urbana hipercultista (G. Schmidt 1962, pp. 26467). En ese caso, apud/apor/apur procederían de *apor o *ap- $r^{14}$, pudiendo ambas retrotraerse a ${ }^{*} \mathrm{H}_{2}$ epor $/{ }^{*} \mathrm{H}_{2}$ ep $-r$. Y apor provendrá a su vez de ${ }^{*} \mathrm{H}_{2} \mathrm{epH} \mathrm{H}_{3} \mathrm{er}$ o ${ }^{*} \mathrm{H}_{2} \mathrm{pH}_{3} \mathrm{er}$. La evolución de adv. de alejamiento a prep. de cercanía no es clara. Por otra parte, pueden existir paralelos en lat. aprilis 'abril' y en apricus 'soleado', que sería una formación típica de adv. y * $H_{3} k_{\sim}^{u}$ 'ojo' (cf. Hamp, 1973), en este caso sobre una base adverbial en $-r$ alargada a su vez por $-i$.

Het. appan adv. 'detrás, después' = luvc. appan, luvj. *apan, lic. epñ, lid. fền-. Ahet. appan puede ir con gen.; o un pron. posesivo que en apariencia es un ac. concordante con el adv. (sólo en copias nhet.). En ahet. sólo una vez con pron. personal en gen.; posp. de dat. (para Starke «aposición partitiva») 'tras, después de', no en ahet. / luvj. *apara- 'posterior' (sólo lo he encontrado en $H E D$ s. $u$. appa y appasiwatt-), luvc. apparanti-15 'el futuro'; lic. epri'posterior, siguiente' ( $i b$.).

La cuestión de appa, appan y sus correlatos ha girado en general en torno a su adscripción a ie. *opi o *apo. La atribución a *opi es la que ha encontrado más aceptación. Y así, $H E G$ (s. u. appa), EHS, p. 352; Gusmani (1961, p. 48 y n. 2), que añade tranquilamente lit. $p \bar{o}$, lat. post, esl. po- como variantes de

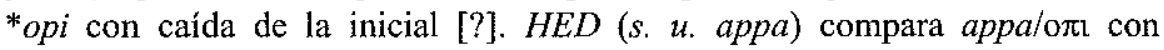
kattalkatti; Morpurgo (1981, pp. 303-307) parte de *op-, variante apofónica de *ep-, de la que app $a(n)$ es un caso en anat. (appa direct., appan ac.), como *olep-i es, p. ej., el loc.

Otros vuelven a la idea de la relación con *apo. Así Sturtevant (1933, pp. 73, $75,212)$. Pedersen $\left(1938\right.$, p. 188) ve en la $a$ - de appa y gr. ¿́mó una $* H_{1}$

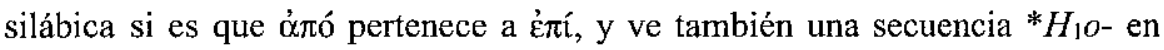
ő $\pi \mathrm{t} \vartheta \varepsilon v$. Hamp (1981, pp. 42-43) rechaza la relación con *e/opi, aunque, admitiendo que parte de los testimonios anat. pueden representar variantes de esta raíz, supone que het. appan puede ser ${ }^{*} H_{1} o p-m$, ac. sg. de su sust. ${ }^{*} H_{1} o p-s$ 'superficie'. Hamp (1965, p. 125) había relacionado appa $(n)$ y sus correspondencias anat. con una base *hapo, donde $* h-\left(H_{4}\right)$ no deja huellas en anat, pero sí en alb., y concretamente en hap < palb. *hápo 'open'. Szemerényi (1955,

conexión'. Otros ven aquí *apo-d, con -d analógica de $p r \bar{o} d$ (cf. $L E W)$. Thurneysen (1904, p. 35) relaciona gr. $\pi \mathrm{o}-\tau_{i}$, av. $p a^{i} t i$.

${ }_{14}$ Reconstruye *apur (como en aaa. abur-) G. Schmidt 1962, p. 267, como ya Johansson 1889 , p. 313.

${ }^{15}$ Para Laroche (1957, pp. 184-85) apparanti- es compuesto de appa y el partic. de ar'llegar'. 
p. 64) hace proceder appa de *apo u *opo, y cree que appan es en cualquier caso una innovación. Dunkel (1982, pp. 84-85 y n. 83) retrotrae appa a *ápo. Appan puede provenir de una forma con $-n$ cuya relación con ai. ápara-recuerda a la de $\dot{\alpha} \gamma \omega \dot{v}$ : $\alpha \dot{\gamma} \varepsilon i ́ \rho \omega$. O tal vez de *op-en, con el recaracterizador de loc. *en. Starke (1977, pp. 133-34) ve en appan el nom.-ac. de un tema en -a (o bien en $-n$ ), en appa el terminativo. Josephson (1966, pp. 134-35, 138) mantiene la identificación de appa con ai. ápa, y niega que appan pueda ser formalmente ac. $\mathrm{n}$.

El adv. *apo expresa probablemente alejamiento desde el exterior de un punto (abl.). Precisamente por ello, anat. común *apa puede haber expresado lo que ocurre 'después', es decir 'a partir de algo'. La suposición de que appa y appan proceden de una forma u otra del tema de *opi prescinde del hecho de que ambas variantes son panat. No sólo no hay un sustantivo *op- en todo el ie. al que atribuir un caso direct. y un ac. semejantes; también el direct. o dat. nominal en $-a$ es relativamente escaso en el resto de las lenguas no het: no existe en lid., tampoco que yo sepa en lic.; en luvc., sólo al parecer en los temas en $-a$ en la forma $-y a$, y en los temas en $-i$ como -iya (Lulahiya). Hay un dat. en $-a$ en luvj. y un caso loc.-direct. $-a$ en pal. Sobre el problema del caso en $-a$ cf. Villar (1981, pp. 18-21). Todo esto puede apuntar a la existencia de un caso en $-a$ antiguo, pero no parece explicar la uniformidad con que en todas estas lenguas se han conservado panat. *apa y *apan. Más bien creo que son variantes de ie. *apo. En el caso de *apa, la base puede ser ${ }^{*} H_{2}{ }_{2} H_{3}-\mathrm{V}$, que explica la falta de notación $h$ - de ${ }^{*} H_{2}$ inicial. La forma subyacente a het. appan, ctc., está igualmente lo bastante extendida como para hacer innecesaria la idea de que es producto de una proporción con anda/andan. El establecimiento de un paralelo con av. apam y ai. apan-indica que anat. *apan proviene de ${ }^{*} H_{2}(e) p$ - o ${ }^{*} H_{2}(e) p_{3^{-}}+-n /$-en. Contiene el alargamiento que aparece en el tema de oblicuo de los heteróclitos en $-r /-n$ y cuyo complementario $-r$ está en el adj. lic. epri-, quizá no en luvc. apparanti-.

\section{6. *(H)up-.}

Ai. upári 'arriba, sobre', nav. upairi/-i, ap. upariy 'arriba; desde arriba', prep. 'sobre' (direct. y loc.; con ac. e instr.), 'pasando sobre' (+ ac. e instr.) / ai. lipa prep. de ac. 'hacia', loc. 'junto a, en, sobre' e instr. 'según, en el curso de', ai. y pāli upan-, av., ap. upa/-ā pref. y prev., prep. de ac. 'hacia, junto a', loc., gen. 'en, junto a'. $K E W$ (s. u. upári) hace proceder ai. upári de ípa 'contra, hacia', con el que puede tener que ver en última instancia como derivado secundariamente antonímico.

Oldenberg (1885, pp. 280-81) reveló la existencia de un prev. upan-en päli vassupanāyika 'fecha en que el monje budista cada año en época de lluvias debe cumplir un período de retiro de 3 meses'. También se encuentra scr. 
upan-áyati en $\breve{S} B$ II, 3, 2, 2 = upagácchati. Sobre esto, J. Schmidt (1885, pp. 281-86) concluye que ai. úpa procede de *up ${ }_{a} n$ como $n \bar{a} m a$ de $n \bar{a} m_{a} n$ y es correspondencia de gr. eol. vं $\pi \alpha$. Upan- constituye un tratamiento *up $n$ V. Persson (1893, p. 236) analiza esta forma como upa-n<*upon/en (de su part. $n \bar{e}, n e, n)$, que es a upár(i) como gót. pan a par. J. Schmidt proporciona una posible clave al aducir como ejemplos paralelos ai. vyapanenti = pāli vapayanti y vinimti $=$ viyanti $(281)$. El autor rechaza un análisis de vyapa-nenti similar al de upan- sobre la base de que no existe sobre *apo sino una formación con final vocálico. En cuanto a vi-n-imti, parece que se deja relacionar con vina $\bar{a}$, pero se pregunta si -in en ai. en final podía dar $-i$, y además en vinä- $n$ podría pertenecer al suf. Por tanto, en ambas formaciones lo probable es una analogía de upan-áyati.

Germ: aing, ofaer, obaer, ofer, as. ovar 'sobre', aaa. ubar, ubir, uber 'sobre, encima', adv. ubiri, upari, dan. asue. over 'sobre', aisl. ofr- 'muy', yfir 'sobre', quizá pn. ubaR (Järsberg), gót. ufar 'sobre', ufarō prep. y adv. 'encima, sobre'. le. *upór es, partiendo de la conservación de la vocal en la secuencia - $V$ - $r$ en final absoluto, la base de pgerm. *ubar, y *upér la de *uber, que representa ${ }^{*} H_{1}$ upér, mientras que i.-ir. *upari, aisl. yfir, aaa. ubiri presuponen *upéri. G. Schmidt (1962, p. 188) compara célt. *upor en airl. for 'sobre', galés gor-. Su suposición de que *upor es contaminación de *upo y *uper es innecesaria (cf. ya Hamp 1953). Por otra parte, la existencia de pcélt. *upor viene confirmada por el testimonio celtib. de una de las inscrs. menores de Peñalba de Villastar, que dice uoramos ednoum, frente a otras de la misma procedencia en que encontramos ueramos, ueramom 'el supremo' (Untermann 1987, p. 69). Es posible igualmente ver *up-r-V en brit. *war-, si no es una deformación de * upor por cuestiones acentuales (Hamp 1972).

Lat. super 'sobre' = gr. i $\pi \varepsilon ́ p$ prep. de gen. 'sobre', ac. de movimiento 'sobre,

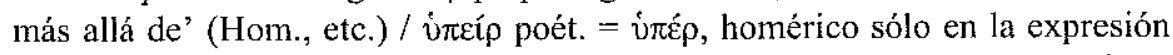

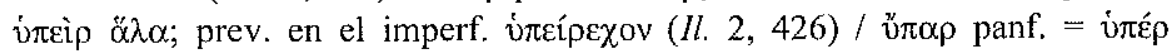

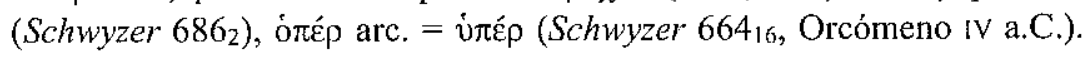

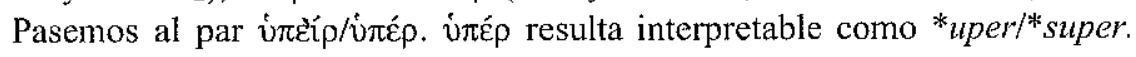

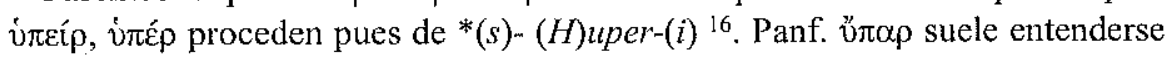

\footnotetext{
16 Respecto a úmeíp, la hipótesis más extendida es la de que se trata de un alargamiento métrico de ijép. Así GEW (s. u. ư⿱氏́), GRD ${ }^{2}$, p. 906 n. 2, Meillet (1893, p. 242) que estima inverosímil que $-i$ final en ie. haya adquirido jamás valor consonántico. Sin embargo, algunos hacen proceder íteíp de úmépl, como Knight (1920, p. 81). Kiparsky (1967, p. 132 y n. 1), que deriva eiv( (l) de éví, basándose en que sólo suelen sufrir alargamiento imputable a razones de metro aquellas palabras que si no de ninguna manera cabrían en él, no aplica

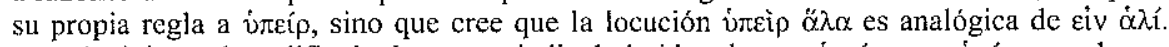
En principio no hay dificultad en prescindir de la idea de que útzíp es = útép con alargamiento métrico. Se observa ese fenómeno en palabras que no caben en el metro en ninguna
} 
como producto de una alteración fonética de vं $x \rho^{17}$. Brixhe (1976, pp. 12 13) observa que, por lo demás, no se encuentra con seguridad paso de timbre $e>$ $a$ en panf., y añade $\pi \varepsilon \rho \tau(\hat{i})$, que viene a invalidar la distinción aventurada por Günther (supra). El autor señala que el hecho de que el fenómeno de apertura se produzca eventualmente en territorio lingüístico dor. no implica una influencia directa y no da mayor importancia a este testimonio. Sin embargo, existe un testimonio adicional que puede indicar que v̋ $\alpha \rho$ refleja un estado de cosas antiguo. Se trata de mic. u-pa-ra-ki-ri-ja (PY An 2981) abl, de un adj. o de un

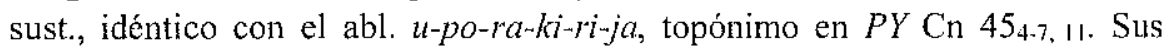

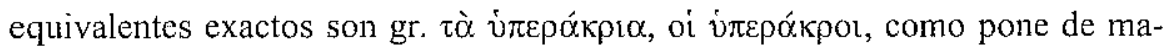
nifiesto Heubeck (1972, pp. 66-67). Ruijgh (1967, p. 175) considera que *upor es la forma original, de la que *upar surgió por asimilación regresiva $o-a>$ $a-a$. Heubeck sugiere una protoforma $* u p r$ en una secuencia fónica $*_{u p r}(r)$-, de modo que un escriba oyó uprakrija y consiguientemente escribió -pa-ra-, mientras que el otro escribió -po-ra-. Ésta es la misma forma base de panf. v̋ঁap. El hecho de que en mic. * $u p r-\mathrm{V}$ siguiera siendo disilábico y no diera $u p r$ - puede ser analógico de casos anteconsonánticos.

Para panf. v้ $\pi \alpha \rho$, mic. u-pa-ra-, u-po-ra-cabe, pues, decir que es probable que representen *upr. En el caso de mic. $u$-po-ra- $u$ - $p a-r a$ - puede pensarse en un interesante doblete ${ }^{*}(s)$ upor ${ }^{*}(s) u p r^{*}$ como en germ, y célt.

En resumen, contamos con tres formas *uper, *upr<* $H_{1} u p-(e) r \mathrm{y} * u p o r$, de las cuales la última es la más problemática por el grado del suf. Ahora bien, sabemos que ${ }^{*} H_{1} u p$ - conllevaba, optativamente, un suf. ${ }_{-} H_{3}$, como se desprende de la sonorización de ${ }^{*}$ - $p$ - ie. que subyace a formas germ. como gót. iup (Hamp 1954), y de formas como gr. i $\pi \alpha(<* u p a$, cf. Heubeck 1972), y tal vez brit. *wa si no $<*$ wo $<*$ upo, así como del mismo ie. ${ }^{*}$ upo $<* H_{1}$ upe $H_{3}-\mathrm{V}$, cuyo g. $\emptyset$ estaría reflejado en $\mathrm{j} \pi \alpha,{ }^{*}$ wa. Por ello reconstruyo alternativamente ${ }^{*} H_{1}$ uper $>*$ uper y ${ }^{*} H_{1}$ up $H_{3}$ er $>*$ upor. La variante ${ }^{*}$ upon $<{ }^{*} \mathrm{H}_{1}$ up $\mathrm{H}_{3}$-en o ${ }^{*}$ upen $<* H_{1}$ up-en se encuentra probablemente en ai. upan-.

circunstancia, lo que no es el caso. Si la expresión v̇iè $\ddot{\alpha}^{2} \alpha \alpha$ estaba congelada como para pasar por las vicisitudes de un tetrasílabo, produciendo vi $\pi \dot{\imath} \rho$ $\alpha \lambda \alpha$, igual puede darse la vuelta al argumento y suponer que, precisamente porque de antiguo esta fórmula era como una sola palabra, - $\varepsilon$ pi - $V$ dio el resultado acostumbrado en interior, donde no había pausa léxica. En manuales y diccionarios se lee que óxép ha sido sustituida funcionalmente por

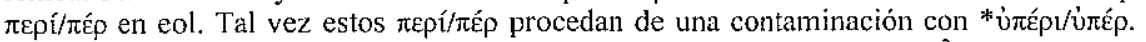

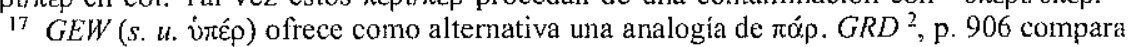

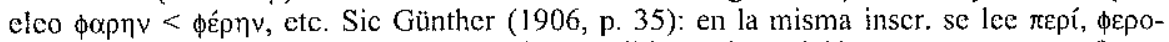
y үर́p $\alpha \zeta$, por lo que este cambio puede circunscribirse a la posición ante consonante, frente

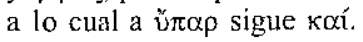




\section{7. *H(e)ndh-.}

Ai. adháh $(-r)$ 'debajo, bajo ( + ac. y gen.)', *adhari- tal vez en adharina $\bar{a}-$ 'despreciado'; nav. $a \delta a^{i} r i$ prep. de ac. y abl. 'bajo', osét. dcelce 'debajo', sog. - $\delta r$ en $c$ ' $\delta r$ 'debajo'. Adj. derivado ai. ádhara-, av. adaro 'el de debajo'.

Germ.: aing. under, aaa. untar, unter, untir, aisl. undir, gót. undar, prep. y adv. 'bajo', aaa. untari, gót. undar $\bar{o}$ 'debajo'.

It: lat. infra 18 'debajo' (Cat., Ter.), inferne 'debajo' (Lucr.), inferus 'inferior', $D E L$ (s. u. inferus) compara gót. undar, ai. ádhara-, observando que $-f$ no es propia del lat. de Roma, sino que es dialectal y responde a los empleos religiosos de inferi, etc. Lo esperable ( $L E W i b$.) seria *inderus. Anat.: Tal vez en lic. etri 'inferior'. Pedersen (1945, p. 50), compara esta forma con ai. ádhara- 'inferior', gót. undar 'bajo'. Para Laroche (1957, p. 183) podría derivarse de annan o forma emparentada y contiene 'el mismo suf. de oposición espacial que het. kattera- $=$ *kat-tera-'.

La adscripción de ai. adháh a ie. *ndhós o *ndhér no queda clara en $K E W$ (s. $u$. adháh) ni en $I E W$, p. 771. En cuanto a i.-ir. *adhari, se ha pensado que tenga $-i$ analógica de upari. Así Wackernagel (1918, pp. 392-93), que habla de una remodelación de *adhas, como germ. undar está construido sobre ufar [!]. Lejeune (1939, pp. 286 87) estima que ai. ádhi procede de ${ }^{*} H_{2} n$ - $d h i$ 'sobre' $\left(<* H_{2}\right.$ en); por el sentido debe separarse de esta forma ap. adiy, que proviene de ${ }^{*} H_{1}$ o-dhi 'en' $\left(<* H_{1} e n\right)$. Por otro lado, sobre un adv. ${ }^{*} H_{1} e n$ 'bajo', homónimo de * $H_{1}$ en 'en' y supuesto a partir de * $H_{1} n i,{ }^{*} H_{1} n e r-$, estarían construidos *ndher, *ndhos, que tal vez reposen sobre ${ }^{*} H_{1} n$-dhi 'bajo' análogo a * $H_{1}$-dhi 'en'. La homonimia explicaría la eliminación de ${ }^{*} H_{i n}$-dhi 'bajo' y la sustitución por formas desarrolladas como ${ }^{*} n d h e r$, igual que $* H_{1}$ en 'bajo' fue sustituido por ${ }^{*} H_{1}$ ner-.

Creo que pgerm. *under $\mathrm{y}$, al menos en parte, ai. adháh, proceden de * $(H)$ ndher, ai. adhari- aaa. untari y aisl. undir tal vez de ${ }^{*}$ Hndheri. ${ }^{*}$ Hndher${ }^{*}$ Hendher-es la única base posible para las formas lat. La forma pura aparece en infer-ne.

$18 L E W$ rechaza con razón la hipótesis de Juret: infra $<*$ ins-rā $<*$ ens como gr. èv y de Thurneysen, que ve ins-en airl. is iss '(por) debajo de', etc. Infr-por *imbr-se sigue de que se relacionaba la secuencia in-inicial con la prep. o la negación in-, p. ej. en intră, y se trataba por tanto la siguiente sílaba como si estuviera en inicial. Pisani $(1931$, p. 67)

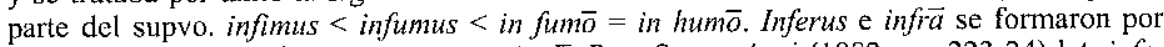
proporción con la serie interus-intimus-intrā. Para Szemerényi (1982, pp. 223-24) lat. inferus < *Hn-dhe-ro-, que contiene el adv/pron. *Han- 'alli, ése' (en *an-yo-s 'otro'), el suf. loc. *dhe y *ro-. Giacomelli (1963) añade una inscr. en una tumba falisca, que dice iffa. En fal. la conservación de ${ }^{*}$-dh- como $f$ - es regular. Por tanto, lat. infiā pudiera ser dialectal, en concreto fal. Si el influjo es de carácter fonético y no léxico, habría surgido por la tendencia a evitar la evolución normal en lat. ante $-r$, que hubiera dado $* i m b r a$, , demasiado distante de *enderos, etc. 
2.8. * ${ }^{*}$ per $H_{3}-$.

Ai. páram está atestiguado desde los Upanisads. Se trata de un nom.-ac- sg. n. de *peros según $G R D^{2}$, p. $872, K E W$ (s. u. paráh). Una respuesta alternativa es la de Reichelt (1901, p. 228) por la que ai. páram, pára, párā son variantes del instr. ie. *prr-em, *prr-m, *per-em, *per-m.

It. Lat. peren-die, o. perom. Sobre peren-die se ha barajado multitud de hipótesis ${ }^{19}$. La explicación de perom y peren-die debe enlazarse con la de lat. per-peram 'mal', supuesto ac. f.: -peram y * perem deben representar un antiguo tema en $-n{ }^{*}$ per $H_{3}-n$, es decir ${ }^{*}$ per $H_{3}-n-\mathrm{V}>$-peram y ${ }^{*}$ per $H_{3}-n-\mathrm{C}>$ peren-. O. perom representa * perom < * per $H_{3}-e n$, igual a ai. páram.

Het. par(r)anda 'más allá de, además de', het. peran/piran adv., prev. y posp. 'ante, delante' = luvc. parran, luvj. *paran. En ahet. con gen. o como posp. de dat. (aposición partitiva). Es loc. frente al valor direct. de para. Zuntz (1936, p. 85) cree que una hipotética relación con gr. $\pi \varepsilon ́ p \alpha v$ reside exclusivamente en el parecido y prefiere conectar piran con para. * pran y * pra estarían en la misma relación que appan y appa, kattan y katta. La vacilación a/i se debería a la costumbre ortográfica o a una diferencia real de la vocal anaptíctica; se apoya sobre todo esta etimología en la comunidad semántica y la no aparición en general de la part. -kan con piran, como con appan, andan, kattan. Oettinger $(1979$, p. 537) reconstruye * perom. Adrados (1975, p. 858) postula *pr-on, y Dunkel (1990, p. 168 y n. 31) piensa en una forma *per-óm, loc. en g. P con la part. enfática de ai. ay-ám, o. ísidum, lat. idem. Neu (1974, pp. 67-68) rechaza la posibilidad de un loc. adesinencial debido a la ausencia de un tema en - $n$ y la no congruencia de peran con un pron. encl. en el mismo caso, y ve un mínimo de dificultad en el postulado de un ac. de dirección

${ }^{19}$ Skutsch separaba *per-en-die 'en 24 horas', que según $L E W$ daría *perindie. Fay reconstruye *per sem $[\mathrm{em}]$ diem. Ehrlich, *peremo- = ai. paramah 'el día más externo'. Strachan (1892, pp. 500-501) propone *peresmi = ai. párasmin. Stolz (1885, p. 498) ofrece *perem $/ *$ porem, que es a ai. páram, o. perom, como lat. idem: o. isidum. Reichelt (1901, p. 228) ve aquí un instr. *prrm/*perm. Osthoff-Brugmann (1910, pp. 351-64) proponen *perno- (u. perne) o *perino-die, y *per(i)no-dino-para peren-dinus. Este puede haber sido lo original, mientras que *per(i)no-dié se constituyó sobre hodie, etc., o bien éste es una construcción independiente, donde die es tal vez, como en hodie, un instr. Para Cupaiuolo (1967, pp. 96-97) *peren- (cf. u. perne, ap. paranam, etc.) es a *pero- como gr. diév a lat. aeuum, un derivado en -en. Perendie sería 'en el día de allá; pasado maranana'. Knight (1920, pp. 76-77) supone que ambos términos no tienen que concordar, y por tanto perem podía ser ya adv. cuando se unió a dié. Peren- puede ser pit. perem $<*$ perm $=$ ai. páram, ac. $m$. del tema per-. Al principio era un adj. con un sust. $m$. sobreentendido. En pit. se usaba ya con el ac. de extensión, de manera que la forma original sería *perendiem. Si o. perum era el ac. de *pero- o per más una part. indef. es incierto. Por lo demás es frecuente que la raiz ${ }^{*}$ per $H_{3}$ - reciba un suf. adverbial ${ }^{*} n$-. Aquí cita $I E W$, p. 811 peren-die con dudas. Deben ser adscritas a este grupo más bien u. perne 'ante' y o. pernúm 'ante' (?) con adv. *n(e) $H_{1}$ $i^{*} n(e) H-m$ como lit. pérnai, ama. vërne, toc. A pärne, B parna (Prósper 1993). 
temático. Según Starke (1977, p. 133), peran es un nom.-ac. n. con función loc., como el resto de los advs. en -n (kattan, appan, andan), al menos desde el punto de vista del het. Se apoya el autor, como es frecuente, en la concordancia visible entre estos advs. y el posesivo encl. -med, -ted, -sed, que suele clasificarse como un ac. n. Josephson (1966) recuerda que nunca se ha demostrado que piran, ser, appa $(n)$, etc., sean de origen nominal. El alargamiento - $n$ en este tipo, concretamente en piran, es de origen adverbial y, en última instancia, pronominal; el autor duda igualmente de que -sed, etc., sean posesivos y no personales regidos, y cree que se trata primitivamente de advs. en $-d$ que tenian en het. las funciones de dat., loc., abl. e instr.

Otten-Souccek (1969, pp. 72-73) han probado que hay dos formas distintas de poses. -smit/-smet 'su de ellos'; la $1 .^{a}$ aparece con sust. en instr./abl. y porta la terminación de instr. nom. -it; la $2 .{ }^{*}$ va con sust. en nom.-ac. n. y advs. Melchert (1984, pp. 122-25) ha realizado una estadística de todos los casos en que un posesivo aparece en ahet: el resultado es de 45 -et: 0 -it con nom.-ac., de 9 -it: 2 -et con instr./abl.). En el caso de los advs. la proporción es de nueve veces - et contra siete ocasiones en que aparece -it. Por ello, nada autoriza la suposición de que peran, etc., sean acs. sg.

Respecto a la etimología de peran, cf. ai. páram, o. perom, lat. peren-die, -peram. En het. peran está representada una protoforma * per $\mathrm{H}_{3}$-en o * per $\mathrm{H}_{3}-n$. Esto significa que el paso -er- -C > -ar- es posterior a la desaparición de $\mathrm{H}_{3}$ ante vocal. Het. parran-da procede de * $\mathrm{pr}_{3} \mathrm{H}_{3}-\mathrm{en}$, posiblemente con geminación del grupo $*_{-r} H$ -

2.9. $* \operatorname{sen} H_{1} \cdots$

I.-ir: aav. hanard, prep. de abl. 'sin'. Etimológicamente tiene relación con ai. sanutár: $G R D^{2}$, p. 894 cree que hanard, perteneciente a la raíz *sen-, *snn- está en la misma relación con ama. sunder, etc. que ai. áparafrente a apatáram. Bartholomae (1889, pp. 16-17,33) cuenta nuestra forma entre el conjunto de locs. en $-r$ y la retrotrae a *sn-er. Beekes (1982, p. 208) parte de ie. *snHér [pero seria posible *senHér o *senHr]. Pinault (1989, p. 43) propone *sénr.

Así, no parece que podamos en este caso contar con la variante anat. en *-n. Sin embargo, se puede plantear una hipótesis hasta ahora no tenida en cuenta. En het, pueden atribuirse a esta raiz formas como sanezziya- 'estupendo (< único, diferente, apartado)', sani, sane, saniya 'en un solo (lugar)'. Para EHS (pp. 246, 348) se trata del dat.-loc. del pron. sani 'uno y el mismo', que conoce un tema en - $a$ en el adv. sannapi sannapi 'aisladamente, aquí y allá, del que a su vez se deriva sannapili- 'solo'. Ahora bien, si uno acepta en cambio que sani es la correspondencia de lat. sine y de toc. A sne, B snai, y teniendo en cuenta que sani, sane-aparece con nasal simple y no geminada, 
cabe replantearse el origen de samnapi. Es evidente que su final es analógico de los advs. pronominales api-va 'alli', kuwapi '(a)dónde'. Por tanto, nada impide pensar que un segmento *sanan-api sufrió síncopa, o que panat. *sann dio directamente sann- ante vocal en una construcción *sann-api congelada en fecha relativamente temprana ${ }^{20}$.

\section{Conclusiones.}

3.1. Existe en ie. una serie de advs. y preps. procedentes de temas nominales que conocemos alargados con diversos sufijos, entre ellos $*_{-r}$ y $*_{-n}$, que a su vez no portan caracterización casual que nos haga pensar que son casos de temas nominales perdidos; p. ej., no hay un elemento añadido ${ }^{*}-e / o s,{ }^{*}-a$, *ai, etc. La única excepción es el caso 'loc.' en *-i, que aparece en *aw(e)ri, *uperi, *ateri y *ndheri, y jamás sobre el tema en *-n. Otra muestra de la inexistencia de flexiones sobre estos temas es el hecho de que estos advs. muestran aleatoriamente una alternancia $\emptyset / \mathrm{P}, \mathrm{P} / \emptyset$, incluso $\emptyset / \emptyset$, que habría que explicar en caso de tratarse de locativos adesinenciales.

3.2. Gran parte de los temas ie. «preposicionales» que conocen un derivado en ${ }^{*} r$ tienen otro paralelo en $*_{\sim n}$ y viceversa, con la visible excepción de ${ }^{*}$ per $H_{3^{-}}$, que exhibe ${ }^{*} n$ en todos los casos, tal vez porque una forma ** perHer era cacofónica o irreconocible y fue tempranamente eliminada.

3.3. La distribución de los sufijos muestra claramente *-r para todo el ie. no anat., * $n$ para el anat., como se ve en el caso de los temas ${ }^{*} H_{2} e u-$, ${ }^{*} H_{1} e n-$, ${ }^{*}$ deu-, ${ }^{*} H_{2} e p-$, posiblemente ${ }^{*} \operatorname{sen} H_{1-}$; la excepción más probable es lic. epri-, êtri-, que por el tardio testimonio de esta lengua (aprox. $\vee$ a.C.) pueden atribuirse a influencia frig., gr. o ir. El i.-ir. se revela como zona de transición, ya que junto a $*_{-r}$ aparece $*_{-n}$ en i.-ir. *upan, *apan.

3.4. Parece claro que estos temas son, cuando menos parcialmente, neoformaciones a partir de temas en laringal a) a la vista de ie. *upor, *apor, donde la coloración de la vocal no se explica satisfactoriamente como adición de *-r a *upo y *apo (que deberían aclararse a su vez) y *perom; b) ante los g. P/P *awer, *ener, *eper, excepto que se acepte que $\varepsilon$ - en las formas descendientes de ${ }^{*}$ ener $\mathrm{y}{ }^{*}$ eper en gr. es producto de la vocalización de ${ }^{*} \mathrm{H}_{\mathrm{L}^{-}}{ }^{21}$, y que ${ }^{*} H$ - inicial tiene como resultado i.-ir. $a$-, lo que es muy dudoso. Pero de no estar formados estos advs. sobre temas en laringal,

20 Obsérvese que nunca se ha podido demostrar que la forma fónica subyacente a $l$-as, l-an sea sama-. Por lo demás, esto tampoco sería imposible si admitimos que sanna- es la tematización del adv. al que nos referimos, y que el añadido analógico es meramente -pi.

21 He dicho más arriba que interpreto $-\alpha$ en final indistintamente como ${ }^{*}-H_{1} / 2 / 3$ en $\alpha F \alpha-$, $\dot{v} \pi \alpha, \dot{\alpha} \pi \alpha-(?)$, y podria añadir casos como $\dot{\alpha} v \dot{\alpha}$ frente a $\alpha$ $v \omega$. Hasta ahora no hay ninguna 
1) habría que ver en los derivados *etero- y *dewero- (?) formaciones tardías en *-ero-, creadas cuando por tematización del adv. aparecieron los adjs. en *-erom, como sobre los advs. loc. en *-ter surgieron los comparativos en *-tero-. Parece oponerse a ello la amplia extensión de *eteros.

2) O bien habría que contar con un proceso generalizado de virddhización secundaria por derivación: en tal caso, habría que partir de tipos $0 / \mathrm{P}:{ }^{*}$ Hter $>$ ${ }^{*}$ Heteros, *dwer $>*$ deweros, ${ }^{*}$ Hwer $>*^{*}$ Heweros, ${ }^{*}$ Hner $>*^{*}$ Heneros, etc. Pero en tal caso no se entiende por qué no iba a ocurrirles lo propio a los advs. de estructura $0 / 0$ (cf. el tipo $* d i w$ - $>*$ deiwos), y tampoco por qué no hay estructura $\mathrm{P} / \mathrm{P}$ en el caso de los derivados *Hndheros y *Huperos.

3.5. El problema que más llama la atención es la caprichosa distribución de $*_{-r}$ y $*_{-n}$. Es sabido que sólo en i.-ir. y anat. existen locs. adesinenciales de temas en $*_{-n}$ (Villar 1981, Neu 1980). Esto podría haber contribuido a la conservación del doblete adverbial en $*_{-r} / *_{-n}$ en i.-ir., donde también aparece -an como loc. puro adesinencial de heteróclitos en $-r /-n$, y a la conservación sólo de advs. en *- $n$ en het., donde existen tagan 'en tierra', taksan 'en medio', pero los heteróclitos tienen invariablemente $-n-i$. El hecho de que los advs. en -an nunca se vieran alargados en $-i$ en ninguna lengua anat. apunta a que en época proto-anatolia ya no existían advs. en $*_{-r} / *_{-} n$ sobre el mismo lexema, ni con diversificación funcional ni ya como alomorfos, que pudiera entenderse que guardan una relación paralela a la que hay entre $*_{-r}$ y $*_{-n} n$ dentro del paradigma. En cambio sí hay dobletes etimológicamente parientes, como appa/appan, para/peran (frente a anda/andan, katta/kattan). Y al revés, que en el resto del ie. y sólo en él aparezcan «locativizaciones» secundarias de * $r$ en * $r-i$ presupone una pérdida muy temprana de los advs. en *- $n$, al menos fuera del i.-ir., donde están de todas formas en franca recesión. Un dato interesante es que los demás advs. nominales en ${ }^{*}-r$ suelen carecer de esta ${ }_{-}-i$, que comparten sin embargo otros advs. primitivos en $*_{-t e r}{ }^{*}$-ter-i (cf. como únicas excepciones para mí conocidas av. ayard: gr. ĩ $\rho-\imath$, het. ser: luvc. sarri). A su vez, el grupo anatolio desconoce el sufijo *-ter-i y en general, al parecer, los advs. con sufijo *-ter (excepto quizá het. katter 'infra' <*km-ter).

Así, se diría que cada una de las dos ramas, anatolio e ie. no anatolio, puede no haber conocido ya como sufijo adverbial predominante sino una de las dos variantes primitivas. Si en ie. común había alguna razón funcional para la

prueba concluyente de la triple representación de laringales en gr. en secuencias $-\mathrm{CH}$, si se exceptúa quizá el caso del dual en $-\varepsilon$. La atribución sistemática de terminaciones en $-\alpha$ a ie. ${ }^{*}-H_{2}$ (Beekes 1969) es naturalmente un razonamiento circular. Existen casos de triple representación en secuencias $H C$ - y $H R C$-, aunque a veces no hay evidencia de que se trate de g. 0,0 , de serlo, puede deberse el timbre resultante a la analogía del $g . P$ correspondiente, que sería idéntico. 
existencia de una doble forma $*_{-r} / *_{-n}$ sobre una misma raiz, ésta tuvo que tender a difuminarse, igual que las posibles diferencias de valor sintáctico del suf. en la heteróclisis ${ }^{*}-r{ }^{*}-n$ se perdieron y quedaron como meros rasgos concomitantes de las marcas desinenciales. En lo que se refiere al i.-ir., es difícil interpretar geográfica o históricamente la idea de que se trata de un área de transición. Sin embargo, es evidente que la división secundaria entre zonas con $*_{-r}$ y con ${ }^{*}-n$ no puede haber sido completamente limpia; restos aislados de la variante descartada tenian que permanecer en ocasiones en algunas zonas. De hecho, en i.-ir. su origen quedó desdibujado completamente en el caso de $*_{-n}>-a, y$ fuera de compuestos aislados sólo quedaría un ejemplo de $*_{-a n}$, en todo caso, en ir. apam.

3.6. No menos enigmática es, en el adverbio, la hipotética distribución original de funciones entre la forma en $*_{-}$, y en $*_{-n} n$ de una misma raíz. Atendiendo al valor de los sufijos en los heteróclitos nominales, y principalmente al valor de $*_{-n}{ }^{22}$, podemos imaginar que ${ }^{*}-r$ como sufijo adverbial expresaba una relación de concordancia y ${ }^{*} n$ una de dependencia; es decir, que ${ }^{*} r$ servía para la atribución (Josephson: «aposición adverbial») como en alemán $\mathrm{Der}$ Mann hier 'el hombre de aquí/que está aquí' y la oración nominal ${ }^{23}$, mientras que ${ }^{*}-n$ se empleaba exclusivamente como circunstancial en oraciones predicativas. El valor indiferenciado en las lenguas ie. ha borrado cualquier posible distinción definitivamente.

Por otra parte, podría especularse con otra posibilidad que difiere algo de la primera: una terminación era empleada para el uso de una forma como prep., la otra para su empleo como verdadero adv. Naturalmente, se objetará que la gran antigüedad de estas formaciones, heredadas por todas las lenguas, nos remiten a una época en que aún no existían las preps. propiamente dichas, como tampoco los procesos comunes de prefijación y preverbación, sino tan sólo adverbios. 'Preposición' tendría que entenderse en este caso como restricción del contenido de un sustantivo que ya aparece de por sí en un caso nocional, de forma parecida a como sucede en anat.: 'en la casa-dentro' (cf. Starke 1977, que lo llama «aposición partitiva»), expresable secundariamente como 'dentro de la casa' por medio de la bifurcación del sistema adverbial en advs. y preps. $Y$ el otro sufijo restringiría el significado verbal: 'vivir-dentro'.

22 Véase en general por extenso Álvarez-Pedrosa, 1988 y R. Adrados, 1991, con diferentes opiniones sobre la consideración funcional de los elementos $*_{-r} *_{-n}$ en la flexión nominal.

23 Es decir, la relación nombre-ađverbio carecería originalmente de cópula como en la oración nominal pura. Cf. el caso del ruso, donde se dice, p. ej., oná - krasiva 'ella es guapa', y esto se aplica exactamente igual a oraciones con adverbio: oná - tam 'ella está alli'. 
De estas explicaciones no se sigue una aclaración de la distribución secundaria de ambos morfemas entre el ie. anat. y no anat., aunque es evidente que la distinción funcional original se borró y el doblete se hizo innecesario. Pero tal vez sí se entienda el porqué de la «locativización» de ${ }^{*}-r$ en ${ }^{*}-r-i$ : el gran grupo que había perdido o dejado de lado ${ }^{*} n$ y perdido igualmente la distinción funcional aneja reorganizó en un momento dado el sistema de distinción de dos clases o funciones sobre un mismo lexema base por medio del morfema de loc., de donde se deduce que en una determinada etapa hubo en ie. no anat. preps. en $*_{-r}$, advs. en $*_{-} r-i$. En ese sentido, es sorprendente como mantenimiento de una tendencia lingüística el testimonio de lit. *aur-en, y el alargamiento de las formas mir. procedentes de i.-ir. *awar.

3.7. Podremos, asimismo, preguntarnos por qué sólo *-er, *-ter, se tematizan formando adjetivos, mientras que no los encontramos terminados en *erios, *-terios < *-eri, *-teri. Pues bien, la conversión del adv. en adj. por el procedimiento corriente de tematización sería producto de la reinterpretación de la secuencia 'el hombre-dentro/que está dentro' (aposición adverbial) como 'el hombre interior' (comprensible en parte a veces como 'el interior del hom-

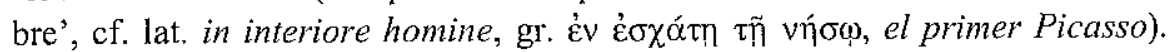
Si ocurrió el fenómeno cuando aún existía el sistema $*_{-r} /^{*} n$, sólo las formas en ${ }^{*} r$ serían susceptibles de tematización, desde la primera de las dos interpretaciones de la variación ${ }^{*}-r / *_{-} n$ que he ofrecido en el parágrafo precedente ${ }^{24}$. En anatolio, al menos del $2 .^{\circ}$ milenio, no se encuentran adjs. en ${ }^{*}$-eros,

24 En otro lugar (1993) dije que «*eno-/*ono-... adopta un carácter especialmente sorprendente en gr. ékeĩvos, an. hinn/hánn, airl. cian 'duradero, lejano'. La reconstrucción *ke-enos, habitual para la forma gr., que tiene diptongo espurio, no explica an. himn ( si no es neoformación sobre $i n n)$, ni air. cian... de *keinos o* $k^{*}$ einos, Estas formaciones carecen de paralelos tipológicos si las analizamos como un adv. (digamos $* k[e] H_{1}-i$, cf. Prósper, en

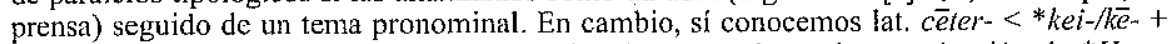
${ }^{*} H_{1}$ el-er-. La conclusión es que el $2 .^{\circ}$ término *eno-puede ser la tematización de ${ }^{*} H_{1} e n-$ 'en'. *k(e) $H_{1}-i-H_{1} e n-,{ }^{*} k(e) H_{1}-i-H_{1} e t-$ podrían concebirse como antiguos sintagmas posposicionales; un paralelo sorprendente existe aún en toc. täpärk, alb. tepër, rus. tepero (con otro segmento inicial $)<* \frac{*}{\text { o-per }} H_{3}(i) »$. Ahora añadiría otra posible interpretación sintáctica: un sintagma como $* k(e) H_{1}-i+H_{1} e n$ puede entenderse como adv. seguido de restrictivo en el sentido de Starke: 'alli, (esto es) dentro', y tematizarse como 'el de allí dentro'. Lógicamente, como se ve en este caso, la tematización afecta a todo el sintagma, y por tanto no me parece tan probable que la tematización de los adjetivos en *-eros derivados de adverbios proceda de una construcción sintáctica de este tipo. Por ejemplo, en het. Éri andan = 'cn casa-dentro', andan, en caso de admitirse una construcción con aposición partitiva, especifica y restringe la noción local de que es portador el morfema de dativo-locativo que porta Éri, razón por la cual se ha interpretado tradicionalmente como posposición. Al estar ambos elementos inmediatamente vinculados entre sí, no puede uno de ellos tematizarse y producir un adjetivo concordante con Éri sin modificar la jerarquía de constituyentes; en todo caso creo que podría sustantivarse: 'en la casa-es decir en el interior', pero ésta me parece una solución poco plausible. En todo caso, entiéndase que la posibilidad de una aposición partitiva antigua no es en sí incompatible con la de una aposición adverbial (cf. 
que deben su existencia a los advs. «preposicionales» en $*_{-r}$. Esto puede interpretarse en el sentido de que la tematización se produjo antes de la escisión del anatolio, culando la división de funciones de ${ }^{*}-r$ y ${ }^{*}-n$ estaba en plená vigencia, y la pérdida posterior de $*_{-r}$ en anatolio contribuyó a que no se conservara el tipo adjetival *-eros. Si, en cambio, sucedió que fue tras la escisión del anatolio cuando surgieron los adjetivos en *aeros en el resto del área indoeuropea, se explica perfectamente, por una parte, que el anatolio no tenga adjetivos en *-eros; por otro lado, en las otras lenguas indoeuropeas el hecho de que sólo se haya tematizado $*_{-r}$ se deberá a la diferencia funcional indicada arriba, si aún quedaban restos suficientemente significativos del sistema original, o simplemente a que ${ }_{-r}$ era ya la terminación predominante y * $n$ ya no existía o estaba en vías de desaparición.

En las lenguas no anatolias, un final ${ }^{*}-r-i$ no existía aún entonces, y ya he dicho que interpreto la variación 'tardía' $*_{-r} / *_{-r-i}$ como correspondiente a preps./advs. en sentido convencional. La función que llamamos 'aposición adverbial' en esta fase relativamente tardía habría sido mayoritariamente adoptada por las formas tematizadas en *-eros en el caso de los adverbios preposicionales que estamos estudiando, $\mathrm{y}^{*}-r \cdot i$, habitualmente en función de complemento del verbo, no sería ya tematizada. En todo caso, estaríamos ante varias tentativas sucesivas, y sucesivamente fracasadas, de conseguir y mantener distinciones morfológicas entre funciones diferentes de una única clase adverbial, y secundariamente entre las clases de palabras «invariables» escindidas de aquélla.

BLANCA PRÓSPER

\section{BiBLIOGRAFIA}

Adrados, F. R. (1975): Lingüística indoeuropea. Madrid.

- (1991): «El formante $-N$ y el origen de la flexión heteroclítica del indoeuropeo», EMERITA 59, pp. 5-21.

Álvarez-Pedrosa, J. A. (1988): La heteróclisis en las lenguas indoeuropeas. (diss.) Oviedo.

Bartholomac, Chr. (1889): "Arisches», $B B$ 15, pp. 1-43.

- (1961): Altiranisches Wörterbuch (2." ed.). Berlin. AIW.

Beekes, R. S. (1969): The development of the Proto-Indoeuropean Laryngeals in Greek. La Haya.

- (1982-83): «On laryngeals and pronouns», $K Z$ 96, pp. 200-232.

Benveniste, E. (1956): «Études sur la phonétique et étymologie de l'ossète», BSL 52, pp. 6-59.

alemán der Mann hier frente a im Koffer drin). Pero me inclino a pensar que la interpretación de las funciones de ${ }^{*}-r$ y ${ }^{*}-n$ como aposición adverbial frente a adverbio dependiente de verbo predicativo es la más probable. 
Brixhe, C. (1976): Le dialecte grec de Pamphilie. Documents et grammaire. Paris.

Brugmann, K. (1904): Die Demonstrativpronomina der indogermanischen Sprachen. Leipzig.

Brugmann, K.-Delbrück, B. (1906): Grundriss der vergleichenden Grammatik der indogermanischen Sprachen $I I^{1}$ (2." ed.). Estrasburgo. GRD ${ }^{2}$.

Bugge, S. (1879): «Etymologische Beiträge», BB 3, pp. 97-121.

Burrow, T. (1955): The Sanskrit language. Londres.

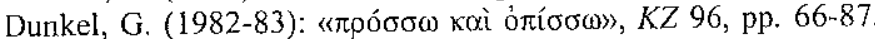

- (1988a): «Laryngeals and particles: ${ }^{*} H_{2} u,{ }^{*} u$ and ${ }^{*}$ awo», Die Laryngaltheorie und die Rekonstruktion des Idg. Laut- und Formensystems, pp. 107-121.

- (1988b): «Indogermanisch *át, Vedisch átha», KZ 101, pp. 53-78.

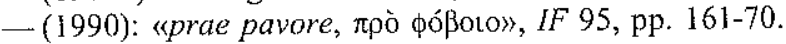

Giacomelli, G. (1963): «La questione del lat. infra e una nuova testimonianza falisca», $A G I 48$, pp. 52-54.

Gusmani, R. (1961): «Il suffisso -tjo- di aggettivi 'locali' e la sua diffusione nelle lingue indocuropee», AION-L 3, pp. 41-58.

- (1963): «Kleinasiatische Miszellen», $I F$ 68, pp. 284-94.

Hamp, E. (1953): "Owclsh guar, Welsh gor-, Bret. gour-, OIr, for-, Gaulish ver-», $B B C S 15$, p. 124.

— (1954): «Gothic iup 'övw'», MLN 69, pp. 39-41.

- (1965): «Evidence in Albanian», Evidence for Laryngeals, pp. 123-41.

- (1972): «British *war and wor-, *wa and wo-», SCelt 7, pp. 135-36.

- (1981): «Indo-european * $\left(H_{e}\right)$ op-», MSS 40, pp. 39-60.

Heubeck, A. (1972): «Syllabic $r$ in Mycenean Greek?», Acta Mycenaea II, pp. 55-79.

Hoffmann, K. (1956): «Notizen zu Wackernagel-Debrunner, Altindische Grammatik $I_{2} \gg, M S S$ 8, pp. 5-24.

Januschas, N. (1962): Das litauische Adverb (diss.). Berlin.

Johansson, K. F. (1889): «Morphologische Studien II», BB 15, pp. 304-16.

Josephson, F. (1966): «Pronominal adverbs of Anatolian: formation and function», RHA 24/79, pp. 133-54.

Knight, C. M. (1920): «Greek and Latin adverbs and their value in the reconstruction of the prehistoric declension»), TCPhS 6, 3, pp. 1-139.

Kronasser, H. (1966): Etymologie der Hethitischen Sprache I. Wiesbaden. EHS.

Laroche, E. (1957-58): «Comparaison du louvite et du lycien», BSL 53, pp. 159-97.

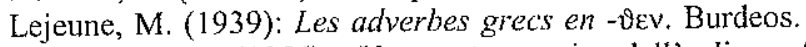

Mastrelli, C. A. (1956): «Un aspetto arcaico dell'eolico», StItFCl 27-28, pp. 272-81.

Mayrhofer, M. (1976): Kurzgefasstes etymologisches Wörterbuch des Altindischen. Heidelberg. $K E W$.

Meier-Brügger, M. (1988): «Zu griechisch ớ $\tau \varepsilon \rho », G l$ 66, pp. 137-42.

Meillet, A. (1893): «Varia», MSL 8, 3, pp. 235-45.

- (1902-1905): Etudes sur l'étymologie et le vocabulaire du vieux slave I-II. París.

Melchert, H. C. (1984): Studies in Hittite Historical phonology. Gotinga.

Morpurgo, A. (1981): «Mycenaean and Greek prepositions: o-pi, e-pi, etc.», Res Mycenaeae, pp. 287-310.

Neu, E. (1974): «Der Anitta-Text», StBoT 18.

- (1980): Der endungslose Lokativ im Althethitischen. Innsbruck.

Nybcrg, H. (1932): «Un pseudo-verbe iranien et son équivalent grec», Symb. Danielsson, pp. 237-61.

Osthoff, F.-Brugmann, K. (1910): Morphologische Untersuchungen VI. Leipzig. 
Oettinger, N. (1979): Die Stammbildung des Hethitischen Verbums. Nuremberg.

Otten, H.-Soucek, V. (1969): «Ein aithethitisches Ritual fürs Königspaar», StBoT 8.

Pedersen, H. (1938): Hittitisch und die anderen indoetropdischen Sprachen. København.

- (1945): Lykisch und Hittitisch. Kobenhavn.

Persson, P. (1893): «Über den demonstrativen Pronominalstamm no-, ne- und Verwandtes», IF 2, pp. 199-260.

Pighi, G. B. (1941): «ll determinativo avverbiale ${ }^{*} R »$, Aevim, pp. 224-35.

Pinault, G. (1989): «Reflets dialectaux en védique ancien», Dialectes dans les litt. indo-aryennes, pp. 35-96.

Pisani, V. (1931): «Miscellanea etymologica», RAL 7 (ser. VI), pp. 65-85.

Pokorny, J. (1959): Indogermanisches etymologisches Wörterbuch. Berna. IEW.

Polomé, E. (1952): «Zum heutigen Stand der Laryngal-Theorie», RBPhH 30, pp. 104152.

Prósper, B. (1990): «Germ. *nēhw- / ai. niçáa, Veleia 7, pp. 377-80.

- (1993): «Estudios sobre la raiz indoeuropea *ne/*no-», $C F C$ n.s. 3.

Puhvel, J. (1984): Hittite etymological dictionary I-II. Berlín/Nueva York (HED).

Rasmussen, J. E. (1987): «Armenian irears 'each other'», LP 28, pp. 46-48.

Reichelt, H. (1901): «Greek $\pi \varepsilon i p \omega$ and $\pi$ pó», $B B$ 26, pp. 223-28.

Ruijgh, C. J. (1967): Études du grec mycénien. Amsterdam.

Schmidt, G. (1962): Studien zum germanischen Adverb (diss.). Berlín.

Schmidt, J. (1885): «Skr. upa, upan», KZ 27, pp. 281-86.

- (1892): «Lateinisch quirquir», KZ 32, pp. 415-19.

Seiler, H. (1957): «Zum prothetischen Vokal im Griechischen: Ablautende 'Präposition' *en/n-1), KZ 75, pp. 1-23.

Stang, C. S. (1966): Vergleichende Grammatik der baltischen Sprachen. Oslo.

Starke, F. (1977): «Die Funktionen der dimensionalen Kasus und Adverbien im Althethitischen», StBoT 23.

Strachan, J. (1892): «Lat. perendie», IF 1, pp. 500-501.

Sturtevant, E. H. (1931): «Hittite etymologies», Lg 7, pp. 1-13.

Szemerényi, O. (1955): «Hittite pronominal inflection and the development of syllabic liquids and nasals», $K Z 73$, pp. 57-80.

- (1982): «Anatolica II», Fschr. Kronasser, pp. 215-34.

Tischler, J. (1977-84): Hethitisches etymologisches Glossar. Innsbruck. HEG.

Untermann, J. (1987): «Lusitanisch, Keltiberisch, Keltisch», IV Col. de lenguas y culturas paleohispánicas, pp. 57-76.

Villar, F. (1981): Dativo y locativo en el singular de la flexión nominal indoeuropea. Salamanca.

Wackernagel, J. (1918): «Indoiranisches», SBAW, pp. 380-411.

Zuntz, L. (1936): Die hethitischen Ortsadverbien arha, para, piran. Múnich. 